\title{
Bilateral controllers for teleoperated percutaneous interventions : evaluation and improvements
}

\author{
L. Barbé, B. Bayle and M. de Mathelin \\ LSIIT, UMR 7005 CNRS-ULP, University of Strasbourg \\ Pôle API, Bd Sébastien Brant, BP 10413, 67412 Illkirch \\ Cedex, France \\ [barbe, bernard, demath] @eavr.u-strasbg.fr
}

\begin{abstract}
This paper presents two teleoperation control schemes developed in the context of percutaneous procedures in interventional radiology. The teleoperation task is characterized by a non linear interaction with the environment. The whole force feedback teleoperation structure is modeled to derive a practical, stable and transparent force feedback. The proposed control approach is based on the adaptation of standard force feedback teleoperation controllers. Position-position and forceposition structures are improved by local compensation loops that include an a priori knowledge of the interactions between the slave robot and the environment made of soft tissues. This contribution allows to improve position tracking capabilities in spite of the nonlinearity of the interaction.
\end{abstract}

\section{INTRODUCTION}

Teleoperation systems are composed of two connected manipulators that enable human operators to perform different tasks in remote, hazardous or delicate environments. Since the first teleoperation system in the 1950's [1], the number and diversity of teleoperation applications have considerably increased. Today, such systems are used in underwater exploration, manufacturing, chemical and biological industry, and, more recently, in the medical field.

In the medical field, the first commercial teleoperation systems were the ZEUS, from Computer Motion and the DaVinci from Intuitive Surgical. They are both dedicated to minimally-invasive surgery (MIS) and specially to laparoscopic interventions. These systems allow the surgeon to perform surgical interventions from a remote place, as illustrated during the Lindberg operation, when Pr. Marescaux carried out the first long distance surgical intervention between New York and Strasbourg on a real patient with a Zeus system [2], [3].

Unfortunately, such teleoperation systems are unilateral and the surgeon only has a visual feedback of the operation field during a teleoperated intervention. In particular, these systems do not allow to feel the interaction forces between the surgical tool and the organs. Yet, it is a critical information for the safety of the patient.

Robotic teleoperation is a very promising technique for the medical techniques that require the protection from X-rays. This is the case of CT-guided percutaneous interventions during which the practitioner performs local treatments directly through the skin of the patient with specific needles. The work flow of such an intervention starts with the planning of the needle trajectory with pre-operative imaging. Even though different imaging techniques are used, we will only consider computed tomography (CT) in this paper. Indeed, in the case of the CT-scan, the quality of the anatomical images is far better than in the case of Ultrasound Imaging (US). Additionally this technique is more convenient and less expensive than Magnetic Resonance Imaging (MRI). During the intervention, the radiologist uses intra-operative CTscan images to obtain the expected needle trajectory. Since imaging is not realtime, the radiologist uses haptic feeling to guide the needle through the anatomical layers, between two CT-scan acquisitions. The success of the intervention mainly relies on the accuracy of the insertion. This requires an intense use of the CT-scan and the radiologist is exposed to a great amount of hermful X-rays.

As this technique offers very interesting possibilities for diagnosis and treatment and since there exists no efficient force feedback teleoperation system for percutaneous treatments, we proposed to develop a teleoperated needle driver with force feedback [4]. Its principle is described on figure 1. Unlike previous teleoperation systems with only visual

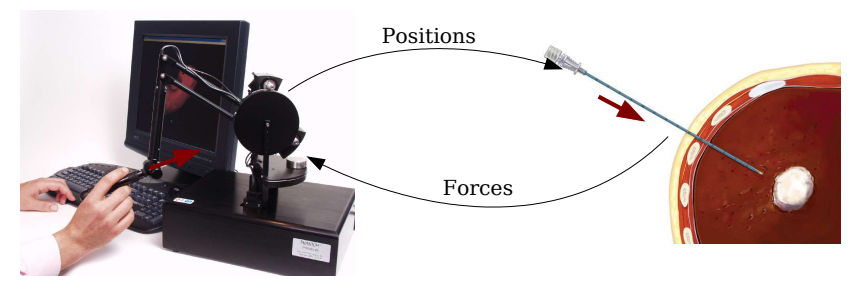

Fig. 1. Principle of the teleoperated needle driver

feedback, this new device will also allow force feedback.

The main problem in force feedback teleoperation consists in the design of the controller that has to be stable and transparent. This paper proposes an evaluation of classical controllers structures in the case of a teleoperated needle insertion in a liver. In the first part of the paper, we address the problem of modeling a teleoperation system in contact with a human operator and an unknown environment. The second part is dedicated to the description of the two main classical control schemes. The third part presents teleoperation simulations were the slave manipulator interacts with a non linear environment, identified from force measurements 
of in vivo needle insertions. We will then conclude on the performance of the evaluated controllers in a teleoperated system dedicated to percutaneous interventions.

\section{SySTEM MODELING}

The most general model to represent a teleoperation system is made up of five blocks: the user, the master manipulator, the bilateral controller, the slave manipulator and the remote environment. This scheme, adapted from [5], is represented on figure 2. The central block (dashed)

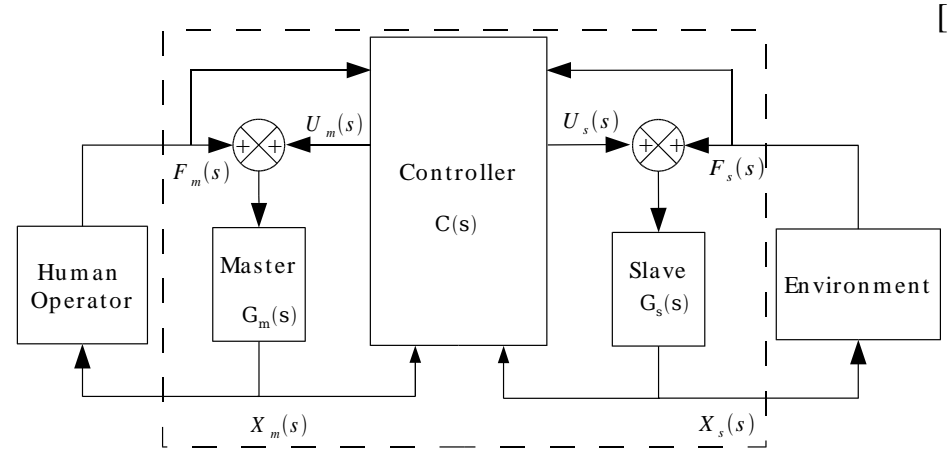

Fig. 2. General model for telemanipulation

represents the master-slave system which interacts with the human operator and the environment.

If we focus on needle insertion tasks, the manipulation requires two degrees of freedom: a translation in the insertion direction and a rotation to orientate the bevel of the needle. However, force feedback is mainly useful for the insertion and the withdrawal of the needle. The orientation of the bevel is only used to bend the needle on a bone, or to extract the needle when some tissue or skin adheres to the needle. So, from now on, we will assume that the interaction forces between the needle and the skin are along the needle shaft and so that the problem has only one degree of freedom.

\section{A. Master and slave manipulators models}

We assume that the actuator nonlinear dynamical effects and the dry friction torque can both be neglected. The dynamic model of the master and the slave manipulators can respectively be expressed in the Laplace domain as:

$$
\begin{aligned}
G_{m}(s) & =\frac{X_{m}(s)}{F_{m}(s)-U_{m}(s)}=\frac{1}{m_{m} s^{2}+b_{m} s}, \\
G_{s}(s) & =\frac{X_{s}(s)}{F_{s}(s)-U_{s}(s)}=\frac{1}{m_{s} s^{2}+b_{s} s},
\end{aligned}
$$

with $G_{m}(s)$ (resp. $G_{s}(s)$ ) the transfer function of the master (resp. the slave) manipulator. The dynamic parameters of the master (resp. the slave) manipulator are its mass $m_{m}$ (resp. $m_{s}$ ), and its viscous coefficient $b_{m}$ (resp. $b_{s}$ ). $X_{m}(s)$ (resp. $X_{s}(s)$ ) represents the position of the master (resp. the slave) manipulator. $F_{m}(s)$ is the force that the human operator applies on the master manipulator and $F_{s}(s)$ the force that the slave manipulator applies on the environment. Finally, $U_{m}(s)$ (resp. $U_{s}(s)$ ) are the forces applied by the actuator driving the master (resp. the slave) manipulator.

\section{B. Human operator model}

The modeling of the human operator is by far the hardest task. The influence of the human operator during a telemanipulation task is very complex. Its modeling requires to take into account:

- the influence of the nervous system responsible for the reflex effects and the time delay to respond to a stimulus;

- the dynamic behavior of the arm.

A meaningful model of the human operator proposed by [6] is shown on figure 3. On this scheme, $M(s)$ is the neural

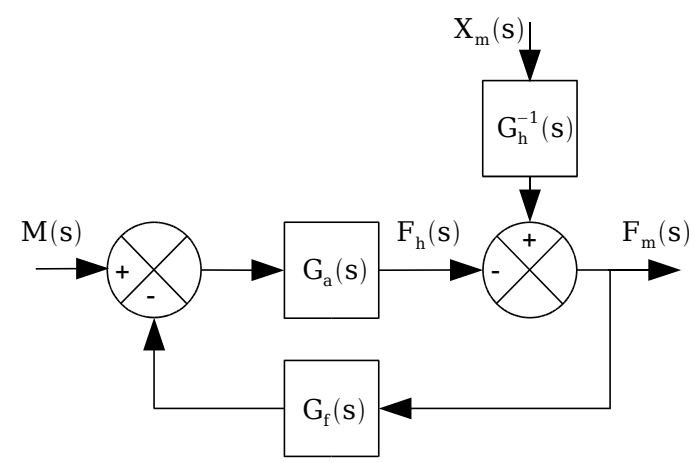

Fig. 3. Human operator model

command. It represents the position of the end-effector of the slave manipulator, as it is desired by the human operator. This information is sent to the muscles by the nervous system, what causes a time lag $T_{d}$. The signal $F_{h}(s)$ is the intended muscle force of the human operator. The transfer function which represents the muscle activation dynamics with the time lag $T_{d}$ is denoted as $G_{a}(s)$ [7]. The transfer function $G_{h}(s)$ corresponds the muscular contraction and to the dynamics of the passive tissues surrounding the joint. It is generally modeled by a second order transfer function. Note that the parameters of all these transfer functions are difficult to obtain because they are different from a human operator to another, and from an application to another. According to the previous notations, the model of the human operator represented on figure 3 is given by:

$$
\begin{aligned}
F_{m}(s) & =F_{h}(s)-G_{h}^{-1}(s) X_{m}(s), \\
F_{h}(s) & =G_{a}(s)\left(M(s)-G_{f}(s) F_{m}(s)\right),
\end{aligned}
$$

with:

$$
\begin{aligned}
G_{h}(s) & =\frac{1}{m_{h} s^{2}+b_{h} s+k_{h}}, \\
G_{a}(s) & =\frac{K_{a} e^{-s T_{d}}}{1+\tau_{a} s}, \\
G_{f}(s) & =\frac{K_{f}}{1+\tau_{f} s},
\end{aligned}
$$

where the parameters of the human operator arm are the mass $m_{h}$, the friction constant $b_{h}$, and the stiffness coefficient $k_{h}$. The time constant for the muscle activation dynamics is $\tau_{a}$. For the neural feedback due to the interaction with the 
master manipulator, the time constant is denoted as $\tau_{f}$. In this model, it is assumed that the master manipulator endeffector and the human operator hand are linked during the manipulation.

For simulation purpose, typical numerical values of the dynamic models of a human operator were chosen:

$$
\begin{aligned}
G_{h}(s) & =\frac{1}{4.04 s^{2}+34.76 s+176.58}, \\
G_{a}(s) & =\frac{176.58 e^{-0.110 s}}{0.025 s+1}, \\
G_{f}(s) & =\frac{0.015}{0.0167 s+1} .
\end{aligned}
$$

\section{Environment model}

Most studies on the design of bilateral controllers are based on a linear model of the environment. In medical applications and particularly in the case of percutaneous interventions, the slave manipulator interacts with a nonlinear environment: the organs, the skin and the bones of the patient. In this paper, we use a realistic nonlinear model that relates forces along the needle shaft direction to the depth of the needle in the organs. It corresponds to a needle insertion in the liver of a pig, at a constant speed, according to the results presented in [8]. Figure 4 presents the in vivo data and the model identified from these data. This model is based

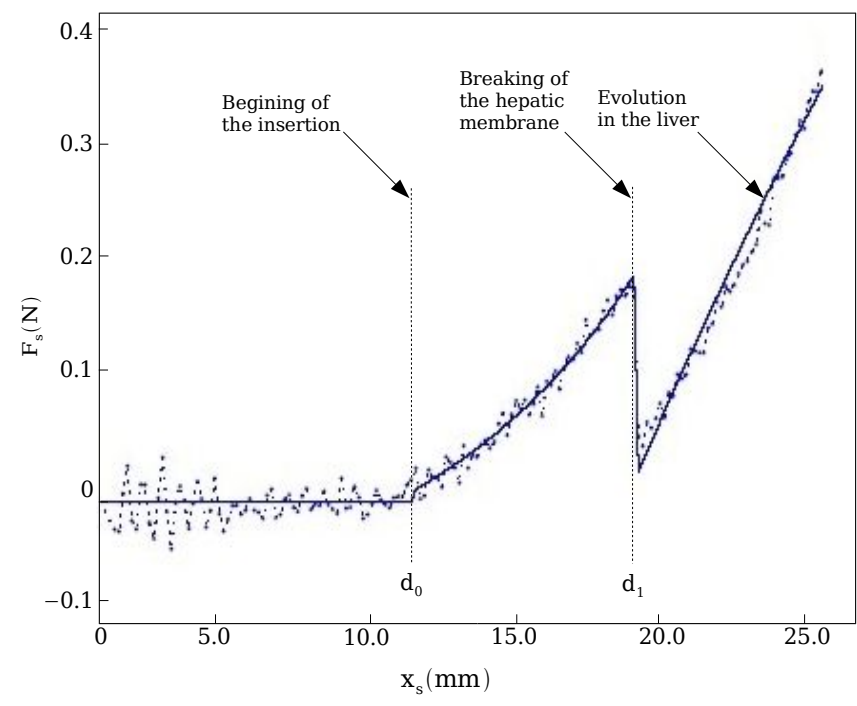

Fig. 4. Needle insertion into a pig liver : measurements and identified model

on the work of Fung [9] for the identification of soft tissues deformations. Besides being nonlinear, it is not continuous, what can be observed at the rupture of the hepatic membrane. So, during the needle insertion phase the data are identified to a set of disjoint functions:

$$
F_{s}\left(x_{s}\right)= \begin{cases}0, & x_{s}<d_{0}, \\ \left(f_{0}+b_{0}\right) e^{a_{0}\left(x_{s}-d_{0}\right)}+b_{0}, & d_{0}<x_{s}<d_{1}, \\ \left(f_{1}+b_{1}\right) e^{a_{1}\left(x_{s}-d_{1}\right)}+b_{1}, & x_{s}>d_{1}\end{cases}
$$

where $d_{0}$ represents the initial position of the skin, assumed to be constant, and $d_{1}$ represents the position of the needle when it breaks the hepatic membrane. The parameters of the model, denoted as $f_{0}, f_{1}, a_{0}, a_{1}, b_{0}$ and $b_{1}$ depend on the mechanical properties of the tissue. For the insertion presented on figure 4 the identified values are $\left[f_{0} ; a_{0} ; b_{0} ; d_{0}\right]=[0.2 ; 0.121 ;-0.098 ; 11.45]$ and $\left[f_{1} ; a_{1} ; b_{1} ; d_{1}\right]=[-3.39 ;-0.031 ; 1.7 ; 19.65]$.

\section{TELEOPERATION CONTROLLERS}

\section{A. Prior works}

The fundamental requirement for any control system is stability. Nevertheless, one of the main objectives of force feedback teleoperation systems is to provide the human operator the feeling that he is directly touching the environment: this property is known as transparency [10]. The bilateral controller which transmits the signals between the master and the slave manipulators has to be designed in order to make the system stable and to offer the optimal transparency performance in spite of time delays, plant disturbances, measurements noise and modeling uncertainties.

Hannaford [11] and Raju [12] works introduced the twoport network representation, based on linear network theory, to analyze the transparency of teleoperation systems and to design specific transparent controllers. In the two-port network context, different representations of the system are possible: hybrid matrix [11], scattering matrix [13] or impedance matrix [14]. To achieve perfect transparency, the hybrid parameters of the two-port teleoperation system have to yield approximatively the identity. Concerning the stability of the two-port network, the problem has often been addressed with the tools of passivity theory [15].

Passivity methods have been extensively applied on systems with time delays [13]. Nevertheless, this criterion is conservative, since it assumes that the human operator and the environment are both passive. To reduce this drawback, some authors used $\mu$-synthesis [16] or unconditional stability to design a stable controller. Recent works on time-domain passivity control [17] have been used to provide stability for a wide variety of environments or human operator motions. These methods, based on an estimate of the dissipation, are still conservative. Indeed, these controllers design techniques do not use models of the human operator or the environment, that should allow to design less conservative controllers.

In the medical field dedicated force feedback teleoperation systems already exist. Most of them are used in laparoscopic MIS [18], [19], [20], [21]. In spite of the lack of realism it may cause, these systems use linear control techniques. As already explained, in the case of needle insertion procedures, the interactions are clearly nonlinear: stiffness may be variable, breaking of membranes occur when the needle is inserted or the needle may touch bones, etc. So, in the rest of this paper, we will introduce interaction nonlinearities in the problem of bilateral control of a teleoperation system using two standard controllers schemes : the Position-Position scheme and the Force-Position scheme. 


\section{B. Position-Position controller}

In this extensively studied control scheme [11], [10], [22], [19], the master position serves as a reference for the slave position controller and, reciprocally, the slave position serves as a reference for the master position controller. This architecture implies that the position controllers of both the master and the slave have good tracking capabilities, since the manipulators have to follow exactly each other.

The Position-Position control scheme corresponding to the general structure of figure 2 is presented on figure 5 . It has a symmetrical structure, except for the $k_{p}$ parameter, that is a position scale factor allowing to change the rate of motion between the master and the slave manipulators.

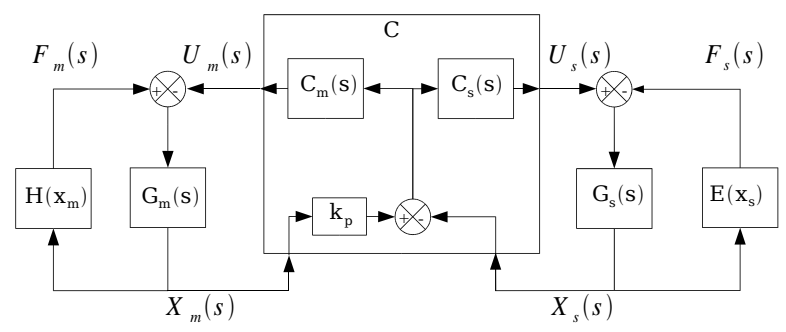

Fig. 5. Block diagram of the Position-Position controller

Force feedback on the master manipulator is the result of the force generated by the master controller, due to the position error tracking when the slave manipulator is in contact with the environment. This control scheme does not require any force sensor. In the literature [10], the position controllers of both the master and the slave manipulators are generally PD controllers. Since there is no integral effect, it is necessary to have high values for the gains of the PD controllers to achieve good tracking. They are tuned once for all, for a given type of environment. If the environment properties vary, this structure may not be adapted. For instance, a sudden contact between the slave manipulator and a hard environment will cause an unstable behavior of the whole system. Therefore, it is impossible to have perfect transparency and robust stability at the same time with this structure.

To reduce the effect of the environment nonlinearity when the hepatic membrane tears, we propose to take an a priori model of the environment into account (see previous section II-C). The model, $\hat{E}$, described by equations (8), allows to estimate the interaction force between the slave manipulator and the environment. From this estimation, we designed a specific controller structure that exhibits a compensation of the nonlinear effects of the environment (see figure 6). This control structure reduces the position error between the master and the slave manipulators, especially during nonlinear phases. The proposed scheme is based on the use of an internal model [23] to increase stability robustness and performances. Indeed, the environment model acts as a feedforward term into the control loop on the slave side and provides a faster force feedback on the master side.

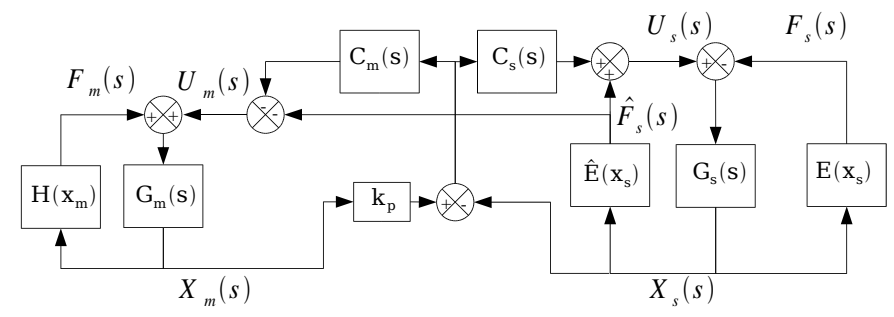

Fig. 6. Block diagram of the modified Position-Position controller, with environment interaction compensation

\section{Force-Position controller}

The Force-Position controller is certainly the most intuitive structure. As illustrated on figure 7, the position of the master manipulator, scaled by a factor $k_{p}$, is used as the reference position for the slave manipulator. The measured forces when the slave is in contact with the environment, scaled by a factor $k_{f}$, are fed-back to the human operator through the master manipulator. Unlike the Position-Position structure,

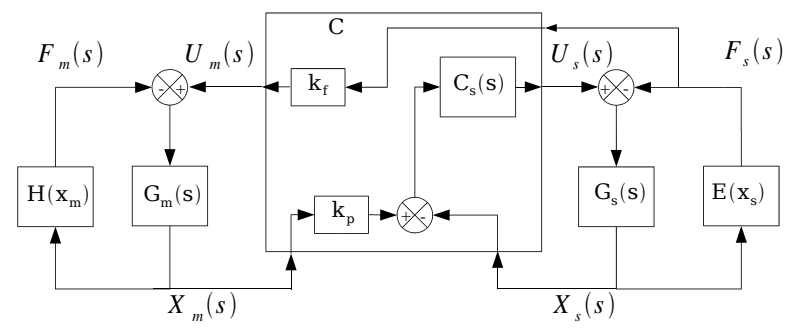

Fig. 7. Block diagram of the Force-Position controller

the Force-Position controller requires a force sensor mounted on the end-effector of the slave manipulator.

It was shown in [24] that this controller is stable for any linear environment if $k_{f}$ is smaller than a critical value. This value is the ratio of the masses of the master and the slave manipulators. The force sensor on the slave manipulator adds noise in the loop that has to be filtered. It usually results a limitation of the servoing bandwidth.

Again, many references in the literature deals with this structure. Most of them are interested in the design of an optimal controller for specific applications such as [19] for medical telesurgery. While many papers are dedicated to transparency and stability performances in linear interaction, to our knowledge there are nearly no study related to non linear interactions as describes in the paper.

Generally, the classical force-position controller does not provide an efficient position tracking. Again, we propose to use an a priori model of the environment in the control law to reduce this disturbance and increase the position tracking capabilities of the controller, similarly to the modified position-position scheme (see figure 6). This a priori model is reduced the disturbance of the environment non linearities. 


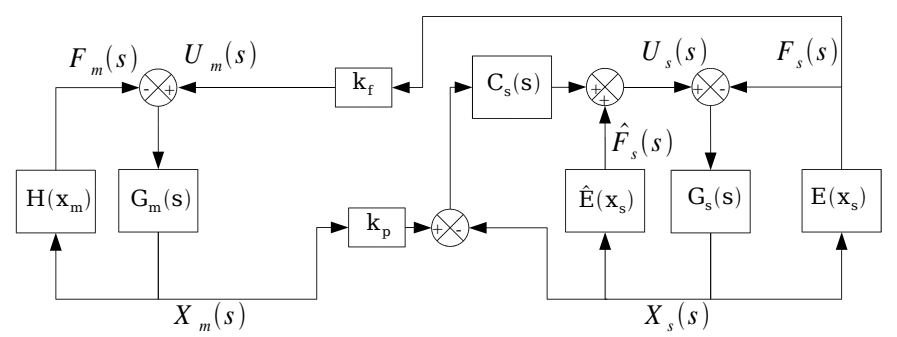

Fig. 8. Block diagram of the modified Force-Position controller

\section{Simulations}

\section{A. System description}

In this section, we evaluate the effectiveness of the modified Position-Position and Force-Position architectures in the case of needle insertions, i.e. in the case of the non linear interaction described by equation (8).

The teleoperation system model for these simulations is the one used by Cavusoglu [19]. It is composed of two identical PHANToM ${ }^{\mathrm{TM}} 1.5$ haptic interfaces, from Sensable Technologies. These device are mechanically constrained so that their end-effector has only one degree of freedom. It move along the vertical direction which is assumed to be orthogonal to the skin. According to equations (1) and (2) the dynamic models can be written as:

$$
G_{m}(s)=G_{s}(s)=\frac{1}{0.09641 s^{2}+2.665 s}
$$

if we suppose that local position control loops compensate the gravity effects.

\section{B. Simulations}

We consider the idealized following task : a human operator desires to reach a point in the liver $35 \mathrm{~mm}$ away from the origin of the needle tip with a constant velocity of $17.5 \mathrm{~mm} / \mathrm{s}$. The entry point on the liver is reached after $11.45 \mathrm{~mm}$, from the initial position of the needle.

1) Position-Position structure: The PD position controllers are as follows :

$$
C_{m}(s)=C_{s}(s)=3.7(s+85.0) ;
$$

They are designed to reach a stability margin of $60^{\circ}$ with natural pulsation of $\omega_{0}=60.3 \mathrm{rad} / \mathrm{s}$, and $k_{p}=1$.

The effects of the non linearities compensation can be observed on figure 9 for position tracking error and on figure 10 for force tracking error. The position tracking error between the master and the slave manipulators are plotted both in the case of the classical position-position controller and the modified position-position controller. When the needle tip is not in contact with the skin, the slave manipulator exactly follows the master manipulator. After the insertion, the controller with the non linearity compensation provides a better position tracking than the classical controller.

After the hepatic membrane puncture, the position error of the classical controller drifts whereas the position error of the modified controller converges to a value below $0.2 \mathrm{~mm}$.

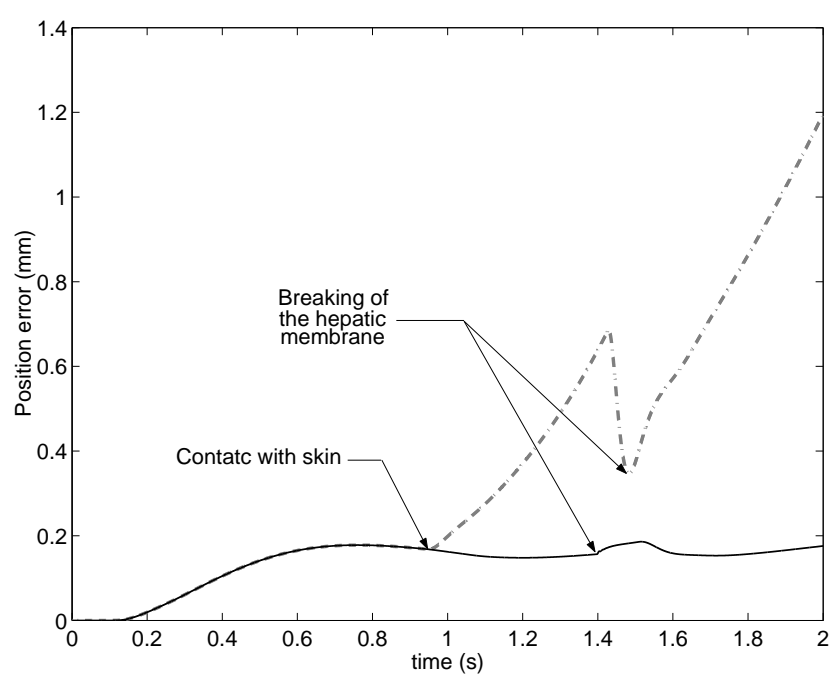

Fig. 9. The position tracking error in classical position-position control scheme (dash) and with non-linear position-position control scheme (solid)

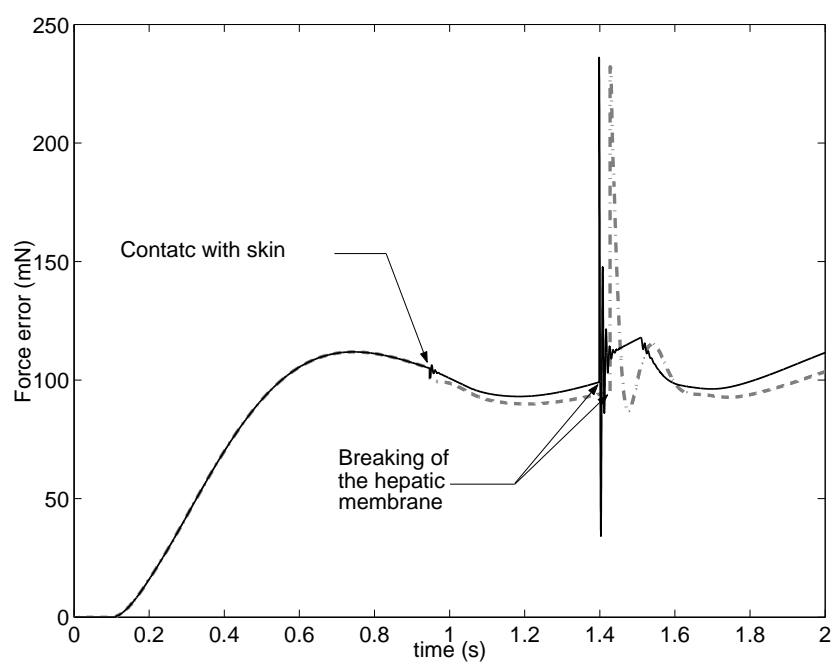

Fig. 10. The force tracking error in classical position-position control scheme (dash) and with non-linear position-position control scheme (solid)

Furthermore the modified controller reduces the transient duration of the force tracking error.

2) Force-Position structure: The slave position controller is the same as the one proposed in the previous scheme, and $k_{f}=1$.

The modified force-position structure provides a better position tracking error than the classical controller (see figure 11). Although the position tracking error of the classical controller drifts with the increasing of the interaction forces, the modified scheme will allow to reach a constant position error without reducing the force tracking performance (see figure 12). 


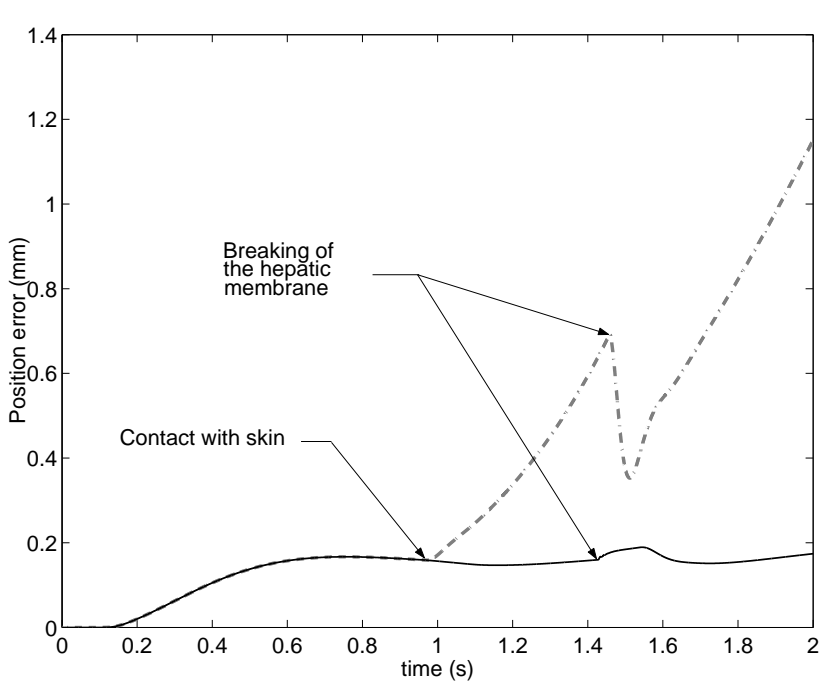

Fig. 11. The position tracking error in classical force-position control scheme (dash) and with non-linear force-position control scheme (solid)

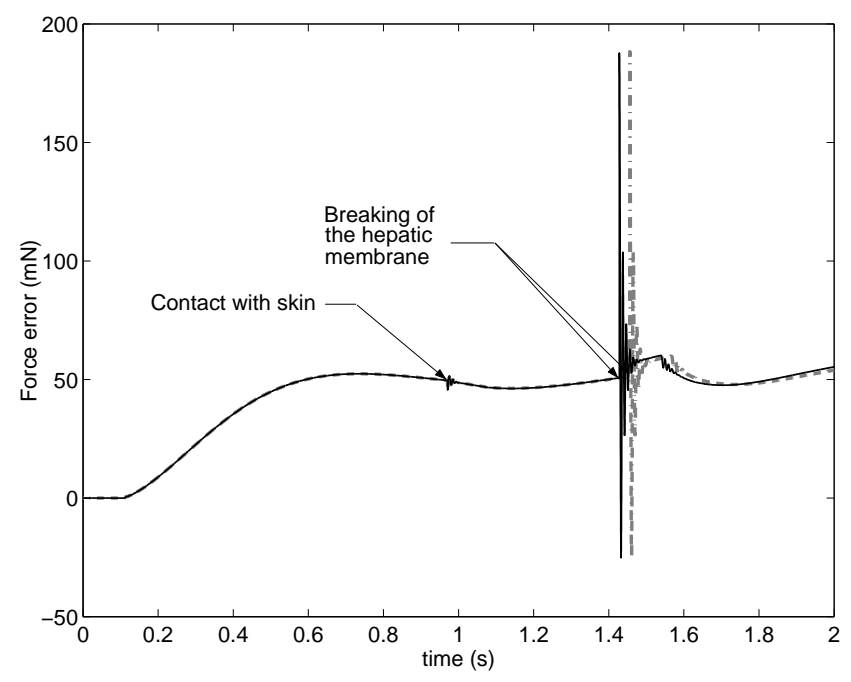

Fig. 12. The force tracking error in classical force-position control scheme (dash) and with non-linear force-position control scheme (solid)

\section{Conclusions}

Classical bilateral teleoperation controllers with PositionPosition and Force-Position structures have seldom been studied in interaction with a non linear environment. In the context of robotized needle insertions non linear interactions are frequent notably because of membrane ruptures. To derive an efficient structure to cope with this problem, we based our study on the use of an interaction model, which was derived from previous experiments. In this paper we illustrated the efficiency of a model based compensation of the non linear effects of the environment. Noticeable improvements in positions tracking have been presented without decreasing force feedback performance. These results are interesting from our application point of view since position tracking is crucial in percutaneous interventions to reach a precise point in the liver.

\section{ACKNOWLEDGMENTS}

This work is supported by the Alsace Regional Council and the French "Centre National de la Recherche Scientifique"(CNRS).

\section{REFERENCES}

[1] R. Goertz, "Electronically controlled manipulator," Nucleonics, vol. 12, pp. 46-47, November 1954.

[2] J. Marescaux, J. Leroy, M. Gagner, F. Rubino, D. Mutter, M. Vix, S. Butner, and M. Smith, "Transatlantic robot-assisted telesurgery," Nature, no. 413, pp. 379-380, 2001.

[3] M. Ghodoussi, S. Butner, and Y. Wang, "Robotic surgery - the transatlantic case," in IEEE International Conference on Robotics and Automation, vol. 2, pp. 1882-1888, May 2002.

[4] B. Maurin, O. Piccin, B. Bayle, J. Gangloff, M. de Mathelin, L. Soler, and A. Gangi, "A new robotic system for ct-guided percutaneous procedures with haptic feedback," in Computer Assisted Radiology and Surgery 18th International Congress, (Chicago, USA), June 2004

[5] J. Yan and S. Salcudean, "Teleoperation controller design using $h_{\infty}$ optimization with application to motion-scaling," IEEE Transaction on Control Systems Technology, vol. 4, no. 3, pp. 244-258, 1996.

[6] H. Kazerooni and M.-G. Her, "The dynamics and control of a haptic interface device," IEEE Transactions on Robotics and Automation, vol. 10 , no. 4, pp. 453-464, 1994.

[7] F. Zajac, "Muscle and tendon : properties, models, scaling and application to biomechanics and motor control," Critical Reviews in Biomedical Engineering, vol. 17, pp. 359-415, 1989.

[8] B. Maurin, L. Barbe, B. Bayle, P. Zanne, J. Gangloff, M. de Mathelin, A. Gangi, and L.Soler, "In vivo study of forces during needle insertions," in Proceedings of Medical Robotics, Navigation and Visualisatoin Scientifc Workshop, (Remagen, Germany), March 2004.

[9] Y. C. Fung, Biomechanics : mechanical properties of living tissues. second ed., 1993.

[10] D. Lawrence, "Stability and transparency in bilateral teleoperation," IEEE Transaction on Robotics and Automation, vol. 9, pp. 624-637, October 1993.

[11] B. Hannaford, "Design framework for teleoperators with kinesthetic feedback," IEEE Transactions on Robotics and Automation, vol. 5, pp. 426-434, August 1989.

[12] G. Raju, G. Verghese, and T. Sheridan, "Design issues in 2-port network models of bilateral remote teleoperation," in IEEE International Conference on Robotics and Automation, pp. 1317-1321, 1989.

[13] G. Niemeyer and J.-J. Slotine, "Stable adaptive teleoperation," Journal of oceanic engineering, vol. 16, pp. 152-162, January 1991.

[14] T. Sheridan and Staff, "MIT research in telerobotics," in Workshop on Space Telerobotics (JPL, ed.), vol. 2, pp. 403-412, July 1987.

[15] R. Anderson and M. Spong, "Bilateral control of teleoperators with time delay," IEEE Transactions on Automatic Control, vol. 34, pp. 494-501, May 1989.

[16] G. M. H. Leung, A. Francis, and J. Apkarian, "Bilateral controller for teleoperators with time delay via $\mu$-synthesis," IEEE Transactions on Robotics and Automation, vol. 11, no. 1, pp. 105-116, 1997.

[17] J.-H. Ryu, H.-S. Kwon, and B. Hannaford, "Stable teleoperation with time-domain passivity control," IEEE Transactions on robotics and automation, vol. 20, pp. 365-373, 2004.

[18] A. Madhani, Design of teleoperated surgical instruments for minimally invasy surgery. $\mathrm{PhD}$ thesis, Department of Mechanical Engineering MIT, USA, 1998.

[19] M. Çavusogglu, A. Sherman, and F. Tendick, "Bilateral controller design for telemanipulation in soft environments," in IEEE International Conference on Robotics and Automation, (Seoul, South Korea), May 2001 .

[20] M. Tavakoli, R. Patel, and M. Moallem, "A force reflective masterslave system for minimally invasive surgery," in Intelligent Robots and Systems, vol. 4, pp. 3077-3082, IEEE/RSJ International Conference on Robotics and Automation, October 2003.

[21] A. Vilchis, J. Troccaz, P. Cinquin, K. Masuda, and F. Pellissier, "A new robot architecture for tele-echography," IEEE Transactions on Robotics and Automation, vol. 19, pp. 922-926, October 2003. 
[22] Y. Yokokohji and T. Yoshikawa, "Bilateral control of master and slave manipulators for ideal kinesthetic coupling-formulation and experiment," IEEE Transactions on Robotics and Automation, vol. 10, pp. 605-620, October 1994

[23] M. Morari and E. Zafiriou, Robust Process Control. Prentice Hall, Englewood Cliffs, 1989

[24] R. Daniel and P. McAree, "Fundamental limits of performances for force reflecting teleoperation," The International Journal fo Robotics Research, vol. 17, pp. 811-830, August 1998. 